\title{
Controlling the Phase of an Oscillator: A Phase Response Curve Approach
}

Denis Efimov

Abstract-The paper discusses elementary control strategies to control the phase of an oscillator. Both feedforward and feedback ( $P$ and PI) control laws are designed based on the phase response curve (PRC) calculated from the linearized model. The performance is evaluated on a popular model of circadian oscillations.

\section{INTRODUCTION}

Rhythmic phenomena are essential to the dynamic behavior of many physical, chemical and biological systems [1], [2], [3], [4], [5], [6]. The last decades have witnessed a growing interest in the analysis and the synthesis of limit cycles [7], [8], [9]. Global and local stability of limit cycles but also the generation of limit cycle oscillations with large basin of attraction in stabilizable nonlinear systems were widely addressed in the control theory literature (see [10] and references therein).

This paper focusses on a different problem. Any periodic oscillation can be characterized by its amplitude, frequency (or frequencies spectrum), and phase. Several strategies have been proposed to control each of these characteristics [11], [12], [13], [14], [15]. Here, we propose elementary strategies to assign the phase of a large class of nonlinear oscillators. Our control objective is to drive an oscillatory system to track the phase of a reference trajectory evolving at the natural frequency of the system.

While this problem can be found in many applications, it has been motived by biological applications in the study of circadian rhythm. Recent work addressing the phase control using model predictive control includes [16], [17]. In biology, a very common and useful tool for studying the phase shift induced by a particular (brief) input is the phase response curve (PRC) [5], [6]. The purpose of this paper is to develop elementary control strategies based on this specific tool. An independent but closely related idea has been proposed in [18] in the context of a neuronal model.

The organization of this paper is as follows. In Section II, we introduce a biological toy motivating example. Section III provides the phase reduction procedure and the definition of the PRC. PRC control map and PRC-based control strategies are the focus of Section IV. Application of these control laws to the motivating example is considered in Section V.

\section{A motivating exAmple}

A common illustration of phase assignment is the jet-lag that most scientists experience when traveling to conferences.

The authors are with the Department of Electrical Engineering and Computer Science (Montefiore Institute, B28), University of Liège, 4000 Liège, Belgium (e-mails: efimov@montefiore.ulg.ac.be, Pierre.Sacre@ulg.ac.be, R.Sepulchre@ulg.ac.be).

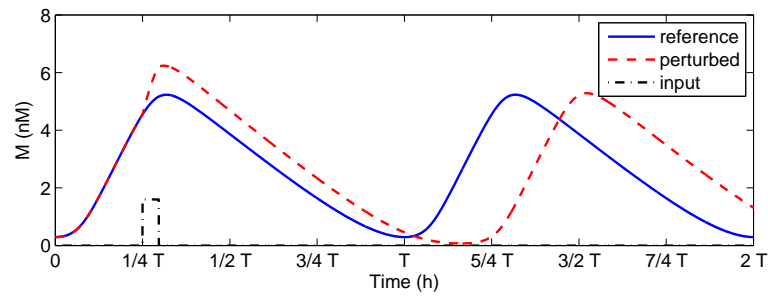

Fig. 1. Illustration of the phase shift caused by a pulse of amplitude $\Delta=1.6$ and duration $T_{w}=1 \mathrm{~h}$ at time $t=T / 4 \mathrm{~h}$ in the Goldbeter model.

The organism needs some time to "reset" the phase of its initial circadian rhythm to shifted environmental light conditions. This problem prompts biologists to study phase resetting and entrainment mechanisms in simple models of circadian oscillations.

The key assumption to the Goldbeter model of Neurospora circadian rhythms is the auto-inhibition of the transcription of the gene frq [19], [21]. This inhibition is through a feedback loop that involves nuclear translocation. Light (modeled by input $u$ ) controls the circadian system by enhancing the rate of $f r q$ gene expression.

Corresponding to these assumptions, one obtains an ordinary differential equation (ODE) system for concentrations as follows:

$$
\begin{aligned}
\dot{M} & =\left(v_{s}+u\right) \frac{K_{I}^{n}}{K_{I}^{n}+F_{N}^{n}}-v_{m} \frac{M}{K_{m}+M}, \\
\dot{F}_{C} & =k_{s} M-v_{d} \frac{F_{C}}{K_{d}+F_{C}}-k_{1} F_{C}+k_{2} F_{N}, \\
\dot{F}_{N} & =k_{1} F_{C}-k_{2} F_{N},
\end{aligned}
$$

where $M>0$ denotes the concentration of frq mRNA, and $F_{C}>0$ and $F_{N}>0$ are used to indicate the concentrations of FRQ in the cytoplasm and in the nucleus, respectively.

The parameters used by Goldbeter are given in Section V. With these parameters, there are limit cycle oscillations (a unique unstable equilibrium and an asymptotically stable limit cycle). If $v_{s}$ is used as a bifurcation parameter, a Hopf bifurcation occurs at $v_{s} \approx 0.60$.

Periodic excitation by light input results in phase and frequency entrainment of the natural circadian oscillations [19], [21], [20]. This means that the application of a suitable input $u$ over a periodic time window close to the natural limit cycle period $T$ may entrain the phase of the system. This phenomenon is illustrated for the specific model in [19], [21]. The input is usually modeled as a sequence of pulses of limited duration and amplitude (for instance, one unique 
pulse of duration $T_{w}=12 \mathrm{~h}$ and amplitude $\Delta=v_{s} / 4$ is applied every 24 hours). Figure 1 illustrates the phase shift resulting from a pulse of duration $T_{w}=1 \mathrm{~h}$ and amplitude $\Delta=v_{s}$. In mathematical biology, the steady state phase shift that results from a particular (brief) input is commonly studied via the phase response curve (PRC) [5], [6].

\section{DERIVING A PHASE MODEL IN THE NEIGHBORHOOD OF A STABLE LIMIT CYCLE}

In this section, we summarize the phase reduction procedure. Starting with a set of ordinary differential equations exhibiting a stable limit cycle, we arrive at a one-dimensional nonlinear phase oscillator model. This phase-reduced model has a single state evolving on the unit circle. Details about this standard procedure can be found in [3], [5], [6], [22].

\section{A. Linearized model}

We consider a (smooth) dynamical system $\Sigma$ defined by differential equations with inputs

$$
\dot{x}=f(x, u)
$$

in which states $x(t)$ evolve on some subset $\mathcal{X} \subseteq \mathbb{R}^{n}$, and input values $u(t)$ belong to some subset $\mathcal{U} \subseteq \mathbb{R}^{m}$ (see [23] for basic definitions and properties regarding such systems). We write $\phi\left(t, x_{0}, u\right)$ for the solution of $\dot{x}=f(x, u)$ with initial condition $x(0)=x_{0}$.

We assume that the "zero-input" system $\dot{x}=f(x, 0)$ has an exponentially stable limit cycle $\Gamma \subset \mathbb{R}^{n}$ with period $T$ and pulsation $\omega=2 \pi / T$. The limit cycle is described by the (non-constant) periodic trajectory $\gamma(t)=\gamma(t+T) \in$ $\mathcal{X}$ for all times $t \geq 0$. This means that the set $\Gamma=$ $\left\{x \in \mathcal{X} \subseteq \mathbb{R}^{n}: x=\gamma(t), t \in[0, T)\right\}$ attracts a non-empty open set of initial conditions in $\mathcal{X}$ and that the linearized system $\Sigma_{*}$ along the trajectory $\gamma(t)$

$$
\delta \dot{x}(t)=A(t) \delta x(t)+B(t) u(t)
$$

with

$$
A(t)=\frac{\partial f}{\partial x}(\gamma(t), 0) \quad \text { and } \quad B(t)=\frac{\partial f}{\partial u}(\gamma(t), 0)
$$

has $n-1$ multipliers strictly inside the unit circle and one multiplier equal to one [24]. Multipliers are the eigenvalues of the monodromy matrix $M=\Phi(T)$ defined for any $A(\cdot)$ via the fundamental solution $\Phi(t)$ associated to the linear equation

$$
\dot{\Phi}(t)=A(t) \Phi(t), \quad \Phi(0)=I_{n}
$$

and the fundamental solution $\Psi(t)$ associated to the adjoint equation

$$
\dot{\Psi}(t)=-A(t)^{T} \Psi(t), \quad \Psi(0)=I_{n},
$$

( $I_{n}$ is the $n \times n$ identity matrix). From those equations, it follows that $\Psi(t)^{T} \Phi(t)=I_{n}$.

Note that the matrix functions $A(t)$ and $B(t)$ are $T$ periodic due to the periodicity of $\gamma(t)$.

\section{B. Phase variables}

Any point $x_{0} \in \Gamma$ can be characterized by a scalar phase $\theta_{0} \in \mathbb{S}^{1}=[0,2 \pi)$, that uniquely determines the position of the point $x_{0}$ on the limit cycle $\Gamma$, because the limit cycle $\Gamma$ is a one-dimensional closed curve in $\mathbb{R}^{n}$ [3], [5], [6]. The smooth bijective phase map $\Theta: \Gamma \rightarrow \mathbb{S}^{1}$ associates to each point $x_{0}$ on the limit cycle its phase $\theta_{0}=\Theta\left(x_{0}\right)$, such that $\phi\left(t, x_{0}, 0\right)=\gamma\left(t+\omega^{-1} \theta_{0}\right)$ if we choose the convention $\Theta(\gamma(0))=0$. The phase variable $\theta: \mathbb{R}_{\geq 0} \rightarrow \mathbb{S}^{1}$ is defined for each trajectory $\phi\left(t, x_{0}, 0\right)$ starting from a point $x_{0}$ on the limit cycle $\Gamma$, as $\theta(t)=\Theta\left[\phi\left(t, x_{0}, 0\right)\right]=\Theta\left[\gamma\left(t+\omega^{-1} \theta_{0}\right)\right]$. Due to the periodic nature of $\gamma(t)$, the function $\theta(t)$ is also periodic. Moreover, the map $\Theta$ can be defined in such a way that $\theta(t)=\omega t+\theta_{0}$ and $\dot{\theta}(t)=\omega$ [5], [6]. That is, $\theta$ evolves linearly in time.

The notion of phase can be extended to any solution $\phi\left(t, x_{0}, 0\right)$ converging to the limit cycle. For an initial condition $x_{0}$ in the basin of attraction of the limit cycle, there exists a unique asymptotic phase $\vartheta_{0} \in \mathbb{S}^{1}$ such that

$$
\lim _{t \rightarrow+\infty}\left|\phi\left(t, x_{0}, 0\right)-\gamma\left(t+\omega^{-1} \vartheta_{0}\right)\right|=0 .
$$

The asymptotic phase map $\Theta: \mathbb{R}^{n} \rightarrow \mathbb{S}^{1}$ maps a point $x_{0}$ in the basin of attraction of the limit cycle to the corresponding asymptotic phase $\vartheta_{0}=\Theta\left(x_{0}\right)$ such that (2) is verified. The asymptotic phase variable $\vartheta: \mathbb{R}_{\geq 0} \rightarrow \mathbb{S}^{1}$ is defined along each solution $\phi\left(t, x_{0}, 0\right)$ starting in the basin of attraction of $\Gamma$, as $\vartheta(t)=\Theta\left[\phi\left(t, x_{0}, 0\right)\right]$. For the particular solution $\theta(t)=\omega t+\theta_{0}$, we have also $\vartheta(t)=\omega t+\vartheta_{0}$ and $\dot{\vartheta}(t)=\omega$.

The notion of asymptotic phase variable can be extended to a nonzero input $u(\cdot)$ provided that the corresponding trajectory $\phi\left(t, x_{0}, u\right)$ stays in the basin of attraction of the limit cycle for all $t \geq 0$. In this case, the asymptotic phase variable is defined as $\vartheta(t)=\Theta\left[\phi\left(t, x_{0}, u\right)\right], t \geq 0$. Then the variable $\vartheta\left(t^{\prime}\right)$ at an instant $t^{\prime} \geq 0$ evaluates the asymptotic phase of the point $\phi\left(t^{\prime}, x_{0}, u\right)$ such that

$$
\lim _{t \rightarrow+\infty}\left|\phi\left(t, \phi\left(t^{\prime}, x_{0}, u\right), 0\right)-\gamma\left(t+\omega^{-1} \vartheta\left(t^{\prime}\right)\right)\right|=0 .
$$

The dynamics of the asymptotic phase variable $\vartheta(t)$ in the case of a nonzero input is hard to derive.

\section{Phase-reduced model}

From the linearized model and the asymptotic phase variable definition, we derive a local phase-reduced model in a small neighborhood of the limit cycle $\Gamma$ for infinitesimal inputs [2], [5], [6].

For a solution of (1) defined in the neighborhood of $\gamma(t)$, the chain rule yields

$$
\begin{aligned}
\frac{d \Theta(x)}{d t}= & \frac{\partial \Theta}{\partial x}\left(\phi\left(t, x_{0}, 0\right)\right) \cdot f\left(\phi\left(t, x_{0}, 0\right), 0\right) \\
& +\frac{\partial \Theta}{\partial x}(\gamma(t)) \cdot B(t) u(t) \\
=\omega & +\frac{\partial \Theta}{\partial x}(\gamma(t)) \cdot B(t) u(t)
\end{aligned}
$$

Defining the infinitesimal phase response curve (iPRC) as

$$
Q(t)=\frac{\partial \Theta}{\partial x}(\gamma(t)),
$$


we obtain the one-dimensional phase equation

$$
\frac{d \vartheta}{d t}=\omega+Q(t) \cdot B(t) u(t)
$$

which is valid (up to the first-order approximation) in a neighborhood of the limit cycle.

Infinitesimal PRC $Q$ serves as a delta-impulse response characteristics in the direction of phase change. It can be computed by solving the adjoint equation: tacking the time derivative of $Q(t) \cdot f(\gamma(t), 0)=\omega$ yields

$$
\begin{aligned}
\dot{Q}(t) \cdot f(\gamma(t), 0) & =-Q(t) \cdot A(t) \dot{\gamma}(t) \\
& =-A(t)^{T} Q(t) \cdot f(\gamma(t), 0) .
\end{aligned}
$$

Thus, the infinitesimal PRC is the solution of the following equation

$$
\dot{Q}(t)=-A(t)^{T} Q(t)
$$

subject to the initial condition

$$
Q(0) \cdot f(\gamma(0), 0)=\omega
$$

For an arbitrary input $u(\cdot)$ that converges exponentially to zero, the phase response curve (PRC) is a map

$$
P R C_{u}: \mathbb{S}^{1} \rightarrow[-\pi, \pi)
$$

characterizing the (asymptotic) phase shift. It is defined as

$$
P R C_{u}(\varphi)=\lim _{T \rightarrow+\infty} \int_{0}^{T} Q\left(\tau+\omega^{-1} \varphi\right) \cdot B\left(\tau+\omega^{-1} \varphi\right) u(\tau) d \tau
$$

When the input $u$ is the Dirac delta function and $B$ is the $i$ th vector of the canonical basis of $\mathbb{R}^{n}$, the PRC map reduces to the $i$ th component of the infinitesimal response curve $Q_{i}(t)$ (up to a change of parameterization $\varphi=\omega t$ ).

\section{PRC-BASED CONTROL DESIGN}

This section presents the main contribution of the paper. It starts with the derivation of the PRC control map. Next, we propose three control strategies: feedforward, proportional (P) feedback and proportional-integral (PI) feedback control.

\section{A. PRC control map}

For control purposes, we consider the application of a fixed input $w(t)$ of finite duration $0<T_{w} \ll T$ at different time instants $t_{0},<t_{1},<\cdots$. The time instants $t_{i}, i \in \mathbb{N}$, are the control parameters. For an initial phase $\varphi$, the input $w(t)$ (asymptotically) causes a phase shift measured by

$$
\operatorname{PRC}_{w}(\varphi)=\int_{0}^{T_{w}} Q\left(\tau+\omega^{-1} \varphi\right) \cdot B\left(\tau+\omega^{-1} \varphi\right) w(\tau) d \tau
$$

The phase difference

$$
\chi=\left(\vartheta-\vartheta_{r}\right) \bmod 2 \pi
$$

between a reference oscillator

$$
\vartheta_{r}(t)=\left(\omega t+\vartheta_{r}(0)\right) \bmod 2 \pi
$$

and a controlled oscillator evolving according to

$$
\dot{\vartheta}=\omega+Q(t) \cdot B(t) w\left(t-t_{0}\right)
$$

thus satisfies

$$
\chi(t)=\chi(0) \text { for } \quad 0 \leq t \leq t_{0},
$$

and

$$
\begin{aligned}
\chi\left(t_{0}+T_{w}\right) & =\chi(0)+P R C_{w}\left(\vartheta\left(t_{0}\right)\right) \\
& =\chi(0)+P R C_{w}\left(\omega t_{0}+\vartheta_{r}(0)+\chi(0)\right) .
\end{aligned}
$$

If the input signal is no longer an isolated application of $w(t)$ but instead a train of such finite-duration inputs

$$
u(t)=\sum_{i=0}^{+\infty} w\left(t-t_{i}\right)
$$

the equations (4)-(5) suggest to study the evolution of the phase difference via the discrete map

$$
\chi_{i+1}=\chi_{i}+P R C_{w}\left(\omega t_{i}+\vartheta_{r}(0)+\chi_{i}\right)
$$

where $\chi_{i}$ denotes the phase error $\chi\left(t_{i}\right)$. This discrete map rests on the assumption that the (asymptotic) phase shift $P R C_{w}\left(\vartheta\left(t_{i}\right)\right)$ has been reached after the (finite) duration $t_{i+1}-t_{i}$. To validate the assumption, one must impose

$$
t_{i+1}-t_{i} \geq T_{s} \quad \forall i \in \mathbb{N}
$$

where the minimal "sampling" time $T_{s}$ is typically chosen according to the limit cycle attractivity.

The discrete map (6) is a first-order discrete-time control system whose control parameters are the pulse timings $t_{i}$, $i \in \mathbb{N}$. Equivalently, one may rewrite (6) as

$$
\chi_{i+1}=\chi_{i}+P R C_{w}\left(\vartheta_{i}\right)
$$

(where $\vartheta_{i}$ denotes the phase $\vartheta\left(t_{i}\right)$ ) and treat $\vartheta_{i}$ as the control variable.

In the remaining section, we discuss three elementary control strategies to select these control parameters: a feedforward control, a proportional feedback control, and a proportional-integral feedback control.

\section{B. Feedforward control of the PRC control map}

The feedforward control strategy is based on the model (6) and does not require any measurement about the current phase of the system. As the phase variable $\vartheta$ evolves on the unit circle $\mathbb{S}^{1}$, phase shifts in both directions can be used to modify the phase.

For the ease of exposition, we assume that PRC has particular properties (it is similar to type II PRC from [25] or type 1 PRC from [1]). The corresponding control strategies for other types of PRCs can be easily deduced from this main case.

Assumption 1: The PRC map is continuous and it has one zero $\theta_{s}^{0} \in \mathbb{S}^{1}$ with negative slope and another $\theta_{u}^{0} \in \mathbb{S}^{1}$ with positive slope, $\theta_{s}^{0}<\theta_{u}^{0}$.

Since the PRC map is $2 \pi$-periodic from (3), the zeros can be arranged in the required order $\theta_{s}^{0}<\theta_{u}^{0}$ changing the initial point on the limit cycle. Define

$$
\begin{gathered}
\theta_{\max }=\arg \max _{\theta \in \mathbb{S}^{1}} P R C_{w}(\theta), \quad P R C_{\max }=P R C_{w}\left(\theta_{\max }\right), \\
\theta_{\min }=\arg \min _{\theta \in \mathbb{S}^{1}} P R C_{w}(\theta), \quad P R C_{\min }=P R C_{w}\left(\theta_{\min }\right),
\end{gathered}
$$


with $\theta_{s}^{0}<\theta_{\min }<\theta_{u}^{0}<\theta_{\max }, P R C_{\max }>0$, and $P R C_{\min }<0$.

The integer part of the numbers

$$
n_{+}=\left(2 \pi-\chi_{0}\right) / P R C_{\max }, \quad n_{-}=-\chi_{0} / P R C_{\min },
$$

determine the number of steps required to drive the initial phase error $\chi_{0}$ into a neighborhood of zero applying positive or negative phase shift, respectively. These numbers are minimal since for their calculation we use the maximum amplitudes of shift $\left(P R C_{\max }\right.$ or $\left.P R C_{\min }\right)$. Defining $N=$ floor $\left[\min \left(n_{+}, n_{-}\right)\right]$, where the function floor $[n]$ returns the greatest integer not bigger than $n$, a natural feedforward control is to apply $N$ pulses of maximal phase shift $(0 \leq$ $i<N)$

$$
\vartheta_{i}=\left\{\begin{array}{ll}
\theta_{\max }, & \text { for } n_{+} \leq n_{-} \\
\theta_{\min }, & \text { for } n_{+}>n_{-}
\end{array} .\right.
$$

A last pulse is needed to annihilate the residual error. The corresponding phase $\vartheta_{N}$ is thus the solution of the following equation (obtained by annihilating the phase error $\chi_{N+1}$ in (7))

$$
\vartheta_{N}: \begin{cases}P R C_{w}\left(\vartheta_{N}\right)+\chi_{N}=2 \pi, & \text { for } n_{+} \leq n_{-} \\ P R C_{w}\left(\vartheta_{N}\right)+\chi_{N}=0, & \text { for } n_{+}>n_{-}\end{cases}
$$

Following this control strategy, the phase error evolves as

$$
\chi_{i}=\left\{\begin{array}{ll}
\chi_{0}+i P R C_{\max }, & \text { for } n_{+} \leq n_{-} \\
\chi_{0}+i P R C_{\min }, & \text { for } n_{+}>n_{-}
\end{array},\right.
$$

for $0 \leq i \leq N$ and we have $\chi_{N+1}=0(=2 \pi)$.

The sequence of phases $\vartheta_{i}, 0 \leq i \leq N$, determines the sequence of times $t_{i}$ as follows: $t_{0}$ is chosen as the first $t \geq 0$ such that

$$
\vartheta_{0}=(\vartheta(0)+\omega t) \bmod 2 \pi
$$

For $i=0, \ldots, n$, one assumes

$$
\vartheta\left(t_{i}+T_{s}\right)=\left(\vartheta_{i}+P R C_{w}\left(\vartheta_{i}\right)+\omega T_{s}\right) \bmod 2 \pi .
$$

Since

$$
\dot{\vartheta}=\omega, \quad \text { for } \quad t_{i}+T_{s} \leq t \leq t_{i+1},
$$

one defines $t_{i+1}$ as the first time $t \geq t_{i}+T_{s}$ such that

$$
\vartheta_{i+1}=\left(\vartheta\left(t_{i}+T_{s}\right)+\omega\left(t-\left(t_{i}+T_{s}\right)\right)\right) \bmod 2 \pi .
$$

This strategy is called "feedforward" since it does not require any measurement of the phase variable.

\section{Proportional feedback control of the PRC control map}

The proportional feedback control strategy assumes online measurements of the current phase variable after each "pulse" application. To realize this strategy it is enough to replace in (13) the values $\vartheta\left(t_{i}+T_{s}\right)$ computed from (12) with measurement values. By measurements we mean the calculation of the phase based on measurements of the state vector $x\left(t_{i}+T_{s}\right)$. The phase of $x(t)$ can be computed using the following algorithm:

$$
\vartheta(t)=\arg \inf _{\vartheta \in \mathbb{S}^{1}}\left\{\left|x(t)-\gamma\left(\omega^{-1} \vartheta\right)\right|\right\}
$$

Of course the application of (14) is valid only in the neighborhood of the limit cycle.

The overall strategy for proportional feedback control is similar to the feedforward one. The desired phases $\vartheta_{i}$ are computed by

$$
\begin{gathered}
n_{+}^{i}=\left(2 \pi-\chi_{i}\right) / P R C_{\max }, \quad n_{-}^{i}=-\chi_{i} / P R C_{\min }, \\
\vartheta_{i}= \begin{cases}\theta_{\max }, & \text { for } 1 \leq n_{+}^{i} \leq n_{-}^{i}, \\
\theta_{\min }, & \text { for } 1 \leq n_{-}^{i}<n_{+}^{i}, \\
h\left(\chi_{i}\right), & \text { otherwise }\end{cases}
\end{gathered}
$$

where the function $h(\chi)$ represents a solution of the equation

$$
h(\chi):\left\{\begin{array}{ll}
P R C_{w}(h(\chi))+\chi=2 \pi, & \text { for } n_{+}^{i} \leq n_{-}^{i} \\
P R C_{w}(h(\chi))+\chi=0, & \text { for } n_{+}^{i}>n_{-}^{i}
\end{array} .\right.
$$

The time instants $t_{i}$ are given by (11) and (13).

\section{Proportional-integral feedback control of the PRC con- trol map}

In the previous sections, it was assumed that the PRC is exactly known, but the map (3) is an approximation only valid for infinitesimal inputs. Suppose, that the static uncertainty on the model (7) is modeled by

$$
\chi_{i+1}=\chi_{i}+P R C_{w}\left(\vartheta_{i}\right)+d
$$

where $P R C_{\min }<d<P R C_{\max }$ is an unknown constant additive disturbance. The presence of $d$ results in a steady state error for both the feedforward and the proportional feedback control.

For proportional-integral feedback control, the desired phases $\vartheta_{i}$ are given by a solution of the following equation

$$
P R C_{w}\left(\vartheta_{i}\right):=\operatorname{sat}\left(-\hat{d}_{i}-\kappa \chi_{i}\right),
$$

with

$$
\hat{d}_{i+1}=\hat{d}_{i}+\gamma\left[\chi_{i+1}-(1-\kappa) \chi_{i}\right], \quad \hat{d}_{0}=0 .
$$

The parameters $\kappa$ and $\gamma$ are chosen such that $0<\kappa<1$ and $0<\gamma<1$. The function sat $(\cdot)$ is defined as

$$
\operatorname{sat}(u)= \begin{cases}u & \text { for } P R C_{\min } \leq u \leq P R C_{\max }, \\ P R C_{\max } & \text { for } u>P R C_{\max } \\ P R C_{\min } & \text { for } u<P R C_{\min } .\end{cases}
$$

The time instants $t_{i}$ are given by (11) and (13).

Note that the proportional-integral feedback control is implemented with an anti-wind up compensator.

\section{Application to motivating exAmple}

In this section, we apply our control strategies to the circadian oscillator model presented in Section II. We choose the parameters used by Goldbeter [19] and given (in suitable units $\mathrm{nM}$ or $\mathrm{h}^{-1}$ ) in Table I.

We use a very simple pulse input defined as

$$
w(t)= \begin{cases}\Delta & \text { for } t<T_{w} \\ 0 & \text { otherwise }\end{cases}
$$

with $T_{w}=1$ and with different values of $\Delta$. Fig. 2 represents analytical and numerical PRC. The "analytical" PRC is obtained from (3) while the "numerical" PRC is computed 
TABLE I

Parameter Values

\begin{tabular}{|c|c||c|c|}
\hline Parameter & Value & Parameter & Value \\
\hline$v_{m}$ & 0.505 & $v_{d}$ & 1.4 \\
$v_{s}$ & 1.6 & $k_{s}$ & 0.5 \\
$k_{1}$ & 0.5 & $k_{2}$ & 0.6 \\
$K_{m}$ & 0.5 & $K_{d}$ & 0.13 \\
$K_{I}$ & 1 & $n$ & 4 \\
\hline
\end{tabular}

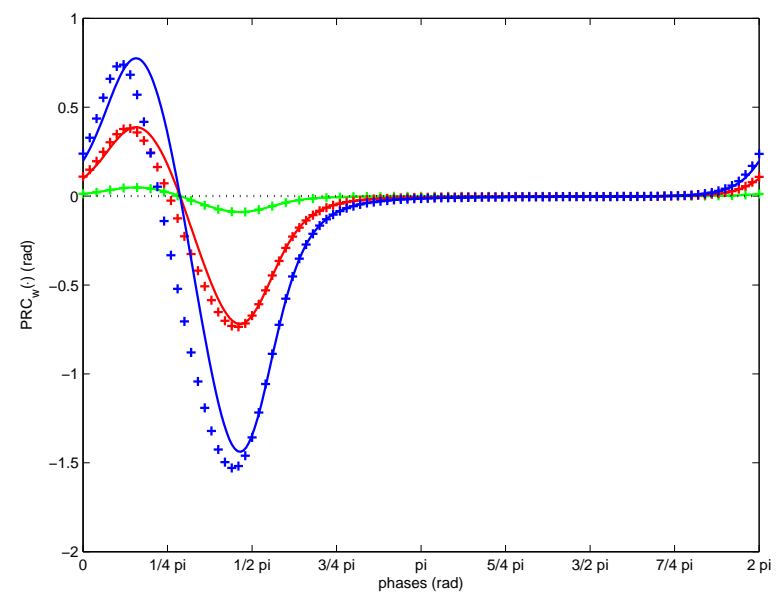

Fig. 2. Analytical (lines) and numerical (cross) PRCs of the Goldbeter model for inputs with $T_{w}=1$ and increasing $\Delta$. The amplitude of the PRC increases with the amplitude of the input (respectively $\Delta=0.1$, $\Delta=0.8$, and $\Delta=1.6$ )

by simulating the nonlinear model. The analytical and the numerical PRCs are very similar for small inputs (the firstorder approximation is valid) but differ for larger inputs.

The simulation results in Fig. 3 are for the input with the largest magnitude $(\Delta=1.6)$. For the control design, we only use the analytical PRC over the domain $\left[\theta_{\max }, \theta_{\min }\right]$. In this region, the phase model has a clear and robust response to the stimulation. We observe the existence of a disturbance $d$ (not exactly constant) between the analytical PRC and the numerical one. The discrete-time evolution of the phase error $\chi$ is shown for four cases: $\chi_{F F}$ is for the feedforward reference (10), $\chi_{O L}$ presents the phase error for the feedforward control, $\chi_{P}$ shows the error of the proportional feedback control, and $\chi_{P I}$ is the error when applying the proportional-integral feedback control strategy. Input curves correspond to the control signal in feedforward, proportional feedback, and proportional-integral feedback control cases (they almost all overlap). The algorithm (14) is used to compute the phase based on measurement of the state vector. The curve $\chi_{F F}$ indicates the reference behavior for the variable $\chi_{O L}$. We choose $T_{s}=T$ such that the discrete model (10) captures the main behavior of the nonlinear model and $\chi_{O L}$ accurately follows $\chi_{F F}$. The phase error $\chi_{P}$ of the proportional feedback control evolves almost as the phase error $\chi_{O L}$. As expected, we observe a steady state error for both the feedforward and the proportional feedback control. The proportional-integral feedback asymptotically reject this constant error.

Figure 4 illustrates the time-evolution of the output vari-

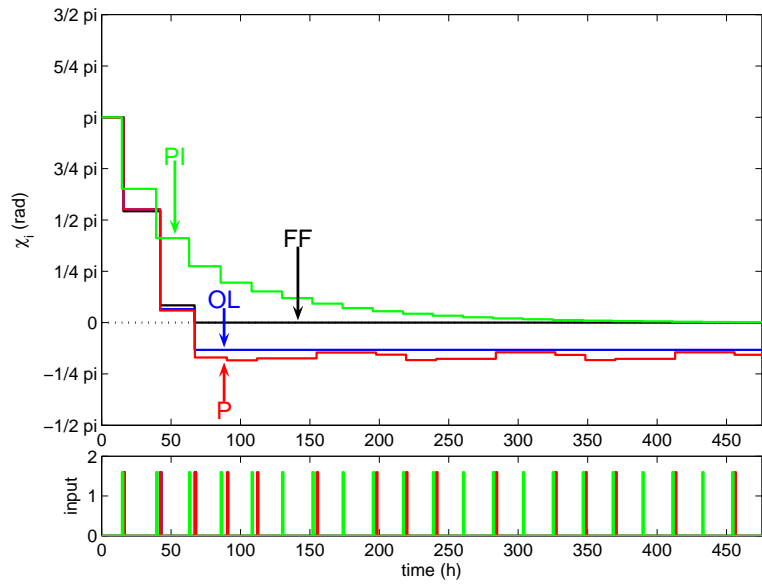

Fig. 3. Discrete-time evolution of the phase error $\chi$ for four cases: $\chi_{F F}$ is for the feedforward reference (10), $\chi_{O L}$ presents the phase error for the feedforward control, $\chi_{P}$ shows the error of the proportional feedback control, and $\chi_{P I}$ is the error when applying the proportionalintegral feedback control strategy $(\kappa=0.25$ and $\gamma=0.9$ ). Input curves correspond to the control signal in feedforward, proportional feedback, and proportional-integral feedback control cases (they almost all overlap).

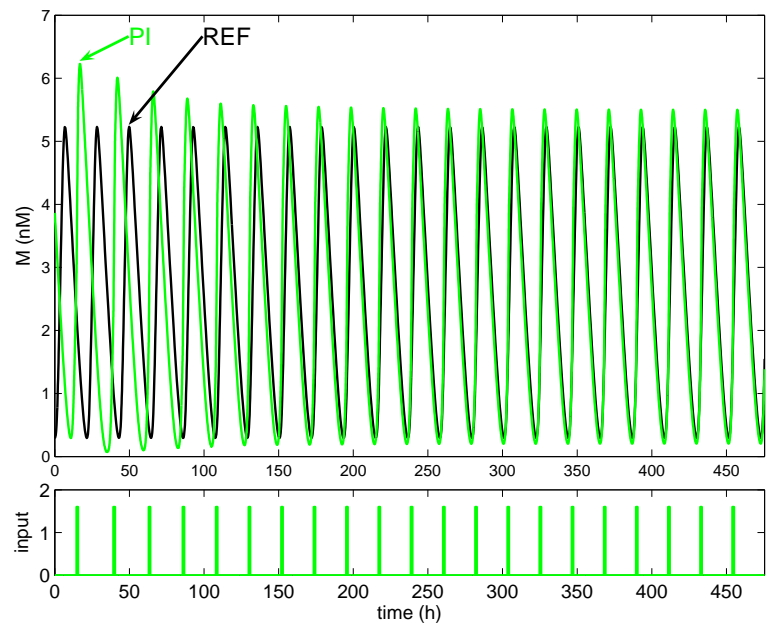

Fig. 4. Time-evolution of the output variable $M$ for the reference oscillator $\left(M_{R E F}\right)$ and for the initially shifted oscillator controlled with the proportional-integral feedback control strategy $\left(M_{P I}\right)$. The input curve corresponds to the control signal in proportional-integral feedback control case.

able $M$ for the reference oscillator $\left(M_{R E F}\right)$ and for the initially shifted oscillator controlled with the proportionalintegral feedback control strategy $\left(M_{P I}\right)$. The timing difference of the maxima in $M$ between those trajectories is a measure of the phase difference for the full-dimensional model. The proportional-integral feedback control strategy (asymptotically) annihilates this difference.

\section{CONCLUSION}

We have presented different control strategies to ensure the convergence of an oscillator's phase to that of a reference phase trajectory with the same natural frequency. The control laws are based on a first-order discrete control system computed from the infinitesimal phase response 
curve of the model. Three control laws were considered: feedforward, proportional feedback and proportional-integral feedback strategies. The control algorithms developed in this paper have been illustrated on the original Goldbeter model of Neurospora circadian rhythm.

The proposed approach is basic but it opens several interesting questions including a formal proof of convergence and its potential use in addressing more challenging engineering questions such as the rendez-vous problem in satellite orbital control.

\section{ACKNOWLEDGMENTS}

This paper presents research results of the Belgian Network DYSCO (Dynamical Systems, Control, and Optimization), funded by the Interuniversity Attraction Poles Programme, initiated by the Belgian State, Science Policy Office. The scientific responsibility rests with its authors.

\section{REFERENCES}

[1] A. T. Winfree, The geometry of biological time. New York: SpringerVerlag, 1980.

[2] Y. Kuramoto, Chemical oscillations, waves, and turbulence. Berlin, Germany: Springer-Verlag, 1984.

[3] I. I. Blekhman, Synchronization in science and technology. New York: ASME Press, 1988

[4] E. Mosekilde, I. L. Mă̌strenko, and D. Postnov, Chaotic synchronization: applications to living systems. River Edge, NJ: World Scientific, 2002

[5] A. Pikovsky, M. Rosenblum, and J. Kurths, Synchronization: A Universal Concept in Nonlinear Sciences. Cambridge University Press, Apr 2003.

[6] E. M. Izhikevich, Dynamical Systems in Neuroscience: The Geometry of Excitability and Bursting. Cambridge, MA: The MIT Press, Nov 2007.

[7] C. Sparrow, The Lorenz equations: bifurcations, chaos, and strange attractors. New York: Springer-Verlag, 1982.

[8] J. Guckenheimer and P. Holmes, Nonlinear oscillations, dynamical systems, and bifurcations of vector fields, 5th ed. New York: Springer, 1997.

[9] A. L. Fradkov and A. Y. Pogromsky, Introduction to control of oscillations and chaos. Singapore: World Scientific, 1998.
[10] G.-B. Stan, "Global analysis and synthesis of oscillations: a dissipativity approach," Ph.D. dissertation, Université de Liège, 2005.

[11] V. K. Astashev, V. I. Babitskiı̌, and M. Z. Kolovskiı̌, Dynamics and control of machines. Berlin, Germany: Springer, 2000

[12] J. Kurths, "A special issue on phase synchronization in chaotic systems," International Journal of Bifurcation and Chaos, vol. 10, no. 11,2000

[13] A. S. Kovaleva, "Frequency and phase control of the resonance oscillations of a non-linear system under conditions of uncertainty," Journal of applied mathematics and mechanics, vol. 68, no. 5, pp 699-706, 2004.

[14] V. Belykh, G. Osipov, N. Kucklander, B. Blasius, and M. Kurths, "Automatic control of phase synchronization in coupled complex oscillators," Physica D-Nonlinear Phenomena, vol. 200, no. 1-2, pp. 81-104, Jan 2005.

[15] D. Efimov, Robust and Adaptative Control of Nonlinear Oscillations. Nauka, 2005.

[16] N. Bagheri, J. Stelling, and F. J. Doyle III, "Circadian phase entrainment via nonlinear model predictive control," International Journal of Robust and Nonlinear Control, vol. 17, no. 17, pp. 1555-1571, 2007.

[17] N. Bagheri, J. Stelling, and F. J. r. Doyle, "Circadian phase resetting via single and multiple control targets," PLoS Comput Biol, vol. 4 , no. 7, p. e1000104, 2008.

[18] P. Danzl and J. Moehlis, "Spike timing control of oscillatory neuron models using impulsive and quasi-impulsive charge-balanced inputs," in Proceedings of the 29th American Control Conference (ACC 2008), Seattle, Washington, USA, Jun 2008, pp. 171-176.

[19] J. C. Leloup, D. Gonze, and A. Goldbeter, "Limit cycle models for circadian rhythms based on transcriptional regulation in drosophila and neurospora." J Biol Rhythms, vol. 14, no. 6, pp. 433-448, Dec 1999.

[20] P. A. Tass, Phase resetting in medicine and biology: stochastic modelling and data analysis, ser. Springer series in synergetics. Berlin: Springer Verlag, 1999.

[21] D. Gonze, J. C. Leloup, and A. Goldbeter, "Theoretical models for circadian rhythms in neurospora and drosophila," C R Acad Sci III, vol. 323, no. 1, pp. 57-67, Jan 2000.

[22] E. Brown, J. Moehlis, and P. Holmes, "On the phase reduction and response dynamics of neural oscillator populations." Neural Comput, vol. 16, no. 4, pp. 673-715, Apr 2004.

[23] E. D. Sontag, Mathematical control theory: deterministic finite dimensional systems, 2nd ed. New York: Springer, 1998, vol. 6.

[24] V. A. Yakubovich and V. M. Starzhinskiǔ, Linear differential equations with periodic coefficients. New York: Wiley, 1975.

[25] D. Hansel, G. Mato, and C. Meunier, "Synchrony in excitatory neural networks," Neural Comput, vol. 7, no. 2, pp. 307-337, Mar 1995. 